
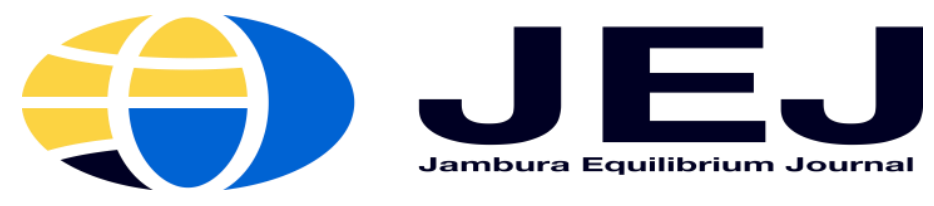

Volume 2. Issue 1. January 2020

P-ISSN 2655-9110

E-ISSN 2656-0445

http://ejurnal.ung.ac.id/index.php/equij

\title{
Spatial Dimensions of Economic Growth, Redistribution, and Poverty Reduction During the Yudhoyono Period in Indonesia
}

Witri Mukti Aji

Master of Economic Planning and Development Policy, Faculty of Economics and Business, Universitas Indonesia

Abstract. This research explores the spatial dimensions of economic growth, redistribution, and poverty reduction in Indonesia during the Susilo Bambang Yudhoyono period (i.e., from 2004 to 2014) using the poverty decomposition method, the growth incidence curve, and several pro-poor growth indices. I gathered my data from the annual National Socio-economic Surveys conducted in Indonesia between 2004 and 2014. Analyzing this data, my thesis presents three key economic insights about the Susilo Bambang Yudhoyono period:1) poverty incidence significantly declined between 2004 and 2014,2) the economic growth that occurred during this period was generally not pro-poor, made evident by an upward sloping growth incidence curve, and 3) regional differences exist in the shape of the growth incidence curve; the pro-poorness of economic growth therefore varies between provinces. Using the classification system proposed by Kakwani and Pernia (2000), I classify provinces into the following five groups with respect to their pro-poor growth index (PPGI). Our empirical results support the pro-poor growth in a nation. However, some provinces such as North Maluku, Gorontalo and Bengkulu experienced non-pro-poor growth and weakly pro-poor. To promote the pro-poor growth in all provinces, the governmental supports in infrastructure and human capital development are essential for the above lagged provinces.

Keywords: Household Expenditures; Economic Growth; Redistribution; Poverty Reduction; Spatial Dimensions; Inequality; Poverty Decomposition Method; Growth Incidence Curve; Pro-Poor Growth Indices.

Inequality in Indonesia is rapidly increasing. In 2002, $10 \%$ of the richest people in Indonesia consumed as much as what $42 \%$ of the poorest people consumed; in 2014, that same measure had increased to 54\%. During the 1997 to 1998 Asian financial crisis, although poverty increased sharply, Indonesia's Gini Ratio also fell.
Everyone was affected, but the richest segment of the populace was hit hardest by the crisis. Since then, the ratio has increased from 0.30 points in 2000 to 0.41 points in 2014, the highest level recorded (World Bank, 2016).

Dabla-Norris, et al. (2015) demonstrated that a higher Gini coefficient 
leads to lower and less stable economic growth. When the share of total income owned by the richest $20 \%$ increases by five percentage points, economic growth drops 0.4 percentage points. Conversely, when the share of total income held by the poorest $20 \%$ increases by five percentage points, economic growth increases by 1.9 percentage points. Profit sharing increases for $20 \%$ of the second and third poorest populations, which also increases growth.

A significant level of inequality can decrease economic growth for everyone, especially if those living in poverty are unable to properly invest in their children's improvement. In these conditions, their children remain vulnerable and cannot escape poverty and move into the consumer class. This subsequently leads to economically disadvantaged people being unable to obtain decent jobs, and they remain in the poverty cycle (World Bank, 2016).

However, economic growth is usually correlated with change in expenditure inequality namely redistribution. When economic growth is followed by an increase in inequality, poverty reduction will decrease. Furthermore, redistribution commonly looks harmless in its effect on growth; just in extreme cases is there some evidence that it may have direct negative impacts on growth. Thus, the effects of direct and indirect redistribution - including the effects of growth from lower inequality pro-growth averages. Redistribution that takes from the rich and provides for the poor is probably going to diminish the work supply of both the rich (who are exhausted more) and poor people (to the extent that they get implies tried advantages that lessen motivators to work). Whatever impacts this has on market wages, they areprobably going to be generally balancing to the extent that they influence the two gatherings a similar way (IMF, 2014).

De Silva and Sumarto (2014) investigated the correlation between economic growth, redistribution, and poverty reduction in Indonesia between 2002 and 2012. These researchers used several pro-poor growth concepts and indices to determine whether growth in this period benefited the poor. Furthermore, Timmer (2014) argued that rapid pro-poor growth requires simultaneous, balanced interaction between growth and distribution processes. Influenced by Indonesian experiences, Timmer's (2014) research introduced a pro-poor growth model that encompassed three levels: improving the 
capabilities of the poor; lowering transaction costs in the economy, especially between rural and urban areas; and increasing demand for those goods and services that are produced by the poor.

\section{LITERATURE REVIEW}

Numerous studies (e.g., Datt \& Ravallion, 1992; De Silva \& Sumarto, 2014; Kakwani, 1997; Kakwani \& Pernia, 2000; Kakwani \& Son, 2008; Ravallion \& Chen, 2003; Timmer, 2004) have analyzed the relationship between poverty reduction, economic growth, and income inequality. These researchers studied whether economic growth was pro-poor or not, while also considering the fact that poverty is influenced not only by economic growth, but also by changes in income inequality. They questioned whether economic growth was helpful for the alleviation of poverty prior to making adjustments for income inequality.

In Indonesia, studies of poverty and inequality are abundant because numerous researchers are attracted to studying the nexus between poverty, economic growth, and inequality (e.g., Akita \& Lukman, 1999; Bhattacharyya \& Resosudarmo, 2015; De Silva \& Sumarto, 2014; Miranti, 2010; Suryahadi, Hadiwidjaja,\& Sumarto,
2012; Suryahadi, Suryadarma, Sumarto, 2009; Timmer, 2004; Van Leeuwen \& Földvári, 2016). For example, De Silva and Sumarto (2014) investigated the correlation between poverty, inequality, and economic growth in Indonesia between 2002 and 2012 and utilized several pro-poor growth methods and indices to define growth as pro-poor or not. In order to analyze the dynamics of pro-poor growth, their study applied growth-redistribution decompositions and pro-poorness indices. Their results showed that economic growth profited those households at the top of the expenditure distribution, while the poor received proportionately fewer advantages than the non-poor.

The majority of the studies that were discussed in this literature review focused on the nexus of poverty alleviation, economic growth, and redistribution in Indonesia as a whole. So, I attempt to explore the spatial dimensions of economic growth, redistribution, and poverty reduction in Indonesia, by region and province during Yudhoyono presidential period in 2004 and 2014.

\section{METHODOLOGY}

This study analyzes the effect of economic growth and redistribution on 
poverty reduction over the period from 2004 to 2014 using data from the National Socio-Economic Surveys (Susenas) in 2004 and 2014, which were conducted by the Central Bureau of Statistics. These surveys include information on household expenditure, location of households (urban and rural, regions and provinces) and household size. To estimate the amount of poverty, this study uses household expenditure data. After calculating per capita expenditure by dividing household expenditure by the number of household members, the amount of poverty is estimated by comparing per capita expenditure with the official poverty lines. ${ }^{1}$ Households under the official poverty lines are considered to be poor; thus, the incidence of poverty (or head count ratio) is obtained by dividing the number of households under the official poverty lines by the total number of households. It should be noted that to calculate real economic growth, expenditures in 2014 are converted to expenditures at constant 2004 prices.

To analyze the extent to which economic growth and redistribution have reduced or raised poverty between 2004 and 2014 in Indonesia, this study employs

${ }^{1}$ The official poverty lines are available for urban and rural areas in each province. the method developed by Kakwani (1997). Using this poverty function, the change in poverty between 2004 (year 1) and 2014 (year 2) can be decomposed into the growth effect $(G E)$ and redistribution effect $(I E)$ as follows.

$$
\begin{gathered}
\Delta P=P\left(z, \mu_{2}, L_{2}\right)-P\left(z, \mu_{1}, L_{1}\right) \\
=G E+I E
\end{gathered}
$$

\section{ANALYSIS AND FINDINGS}

It should be noted that there are now 34 provinces in Indonesia, eight of which have been created since 1999 under decentralization, i.e., North Maluku(formerly, Maluku), West Papua (formerly, Papua), Banten (formerly, West Java), Bangka Belitung Islands (formerly, South Sumatra), Gorontalo (formerly, North Sulawesi), Riau Islands (formerly, Riau), West Sulawesi (formerly, South Sulawesi) and North Kalimantan (formerly, East Kalimantan). But, in this study, West Papua, Riau Islands, West Sulawesi and North Kalimantan are merged, respectively, with Papua, Riau, South Sulawesi and East Kalimantan. Therefore, the analysis is conducted using 30 provinces. 


\section{Changes in Poverty}

Table 1 shows changes in the incidence of poverty (i.e., poverty headcount ratio, defined by equation (4)) by region and by province. Indonesia saw a significant decrease in the incidence of poverty in the period, from $27.3 \%$ to $10.0 \% .^{2}$

Table 1. Poverty Headcount Ratio in 2004 and 2014and Change in Poverty Headcount Ratio between 2004 and 2014 (in \%)

\begin{tabular}{|c|c|c|c|c|c|c|}
\hline & $\begin{array}{r}2004 \\
\text { (A) }\end{array}$ & $\begin{array}{l}2004 \\
\text { Rank }\end{array}$ & $\begin{array}{r}2014 \\
\text { (B) }\end{array}$ & $\begin{array}{l}2014 \\
\text { Rank }\end{array}$ & $\begin{array}{l}\text { Absolute change } \\
\text { in poverty headcount ratio }\end{array}$ & $\begin{array}{r}\text { Proportional change } \\
\text { in poverty headcount } \\
\text { ratio }^{(1)}\end{array}$ \\
\hline Province & & & & & $"=(\mathrm{B})-(\mathrm{A}) "$ & \\
\hline Aceh & 26 & 10 & 16.3 & 3 & -9.6 & -4.6 \\
\hline North Sumatera & 18 & 23 & 7.7 & 19 & -10.3 & -8.5 \\
\hline West Sumatera & 26.5 & 9 & 6.6 & 22 & -19.9 & -13.9 \\
\hline Riau & 26 & 11 & 6.6 & 23 & -19.3 & -13.6 \\
\hline Jambi & 19.9 & 21 & 8 & 17 & -11.9 & -9.1 \\
\hline South Sumatera & 24.4 & 13 & 12.7 & 10 & -11.7 & -6.5 \\
\hline Bengkulu & 20.6 & 18 & 16.5 & 2 & -4.1 & -2.2 \\
\hline Lampung & 32.2 & 4 & 13.9 & 7 & -18.4 & -8.4 \\
\hline Bangka Belitung & 18.5 & 22 & 5.7 & 24 & -12.8 & -11.7 \\
\hline Jakarta & 9.1 & 29 & 3 & 30 & -6.2 & -11.2 \\
\hline West Java & 22.1 & 17 & 8.4 & 16 & -13.6 & -9.6 \\
\hline Central Java & 35.4 & 3 & 13.4 & 8 & -22 & -9.7 \\
\hline Yogyakarta & 29.4 & 7 & 11.9 & 12 & -17.5 & -9.1 \\
\hline East Java & 39.1 & 2 & 11.5 & 13 & -27.6 & -12.2 \\
\hline Banten & 14.7 & 27 & 4.8 & 27 & -9.9 & -11.2 \\
\hline Bali & 14.4 & 28 & 3.7 & 29 & -10.6 & -13.4 \\
\hline West Nusa Tenggara & 32 & 5 & 15.8 & 6 & -16.2 & -7 \\
\hline East Nusa Tenggara & 31.6 & 6 & 16.3 & 4 & -15.3 & -6.6 \\
\hline West Kalimantan & 17.5 & 25 & 7.9 & 18 & -9.6 & -8 \\
\hline Central Kalimantan & 20.6 & 19 & 5.4 & 26 & -15.2 & -13.3 \\
\hline South Kalimantan & 16 & 26 & 3.8 & 28 & -12.2 & -14.4 \\
\hline East Kalimantan & 20.1 & 20 & 5.7 & 25 & -14.4 & -12.7 \\
\hline North Sulawesi & 17.9 & 24 & 7.3 & 20 & -10.5 & -8.9 \\
\hline Central Sulawesi & 28.2 & 8 & 12 & 11 & -16.1 & -8.5 \\
\hline South Sulawesi & 25.2 & 12 & 9 & 15 & -16.2 & -10.3 \\
\hline South East Sulawesi & 24 & 14 & 11 & 14 & -13 & -7.8 \\
\hline Gorontalo & 23.4 & 16 & 16 & 5 & -7.3 & -3.8 \\
\hline Maluku & 23.7 & 15 & 13.2 & 9 & -10.5 & -5.9 \\
\hline North Maluku & 4.2 & 30 & 7.2 & 21 & 3 & 5.4 \\
\hline Papua & 49.4 & 1 & 22.9 & 1 & -26.5 & -7.7 \\
\hline Indonesia & 27.3 & & 10 & & -17.3 & -10 \\
\hline \multicolumn{7}{|l|}{ Region } \\
\hline Sumatra & 24 & 4 & 10.2 & 2 & -13.8 & -8.6 \\
\hline Java-Bali & 29.2 & 1 & 9.7 & 4 & -19.4 & -11 \\
\hline Kalimantan & 18.2 & 5 & 5.7 & 5 & -12.4 & -11.5 \\
\hline Sulawesi & 24.1 & 3 & 9.9 & 3 & -14.2 & -8.9 \\
\hline East Indonesia & 28.2 & 2 & 16.9 & 1 & -11.3 & -5.1 \\
\hline Indonesia & 27.3 & & 10 & & -17.3 & -10 \\
\hline
\end{tabular}

${ }^{2}$ The incidence of poverty is estimated using the official poverty lines for urban and rural areas in each province. Our estimate in 2004 at $27 \%$ is much larger than the one reported by the Central Bureau of Statistics $(18 \%)$, but the result of our pro-poor growth analysis will not be changed qualitatively. It should be noted that the headcount ratio of $18 \%$ can be obtained by lowering the official poverty lines by $10 \%$. 
Spatial Dimensions of Economic Growth, Redistribution, and Poverty Reduction During the Yudhoyono Period in Indonesia

\section{Economic Growth}

As discussed in the previous chapter, the change in poverty can be decomposed into growth and redistribution components. It is thus imperative to investigate economic growth and redistribution (i.e., changes in inequality) in the study period. This section will present economic growth by region and province, where economic growth is measured by the growth of mean per capita expenditure. Table 2 shows annual average growth rate of mean per capita expenditure. Over the period from 2004-2014, mean per capita expenditure has increased at an annual average growth rate of 5.5\% in Indonesia.

Table 2. Annual Average Growth Rate of Mean Per Capita Expenditure (in Rupiah) between 2004 and 2014 (at 2004 Constant Prices)

\begin{tabular}{|c|c|c|c|c|c|c|}
\hline \multirow[b]{3}{*}{ Province } & \multirow{2}{*}{\multicolumn{2}{|c|}{ Mean Per Capita Expenditure }} & \multirow{2}{*}{\multicolumn{2}{|c|}{ Growth rate }} & \multicolumn{2}{|c|}{ Population Share } \\
\hline & & & & & & Share \\
\hline & 2004 & 2014 & Growth rate & Rank & 2004 & 2014 \\
\hline Aceh & 202,250 & 259,628 & $2.50 \%$ & 30 & $1.70 \%$ & $1.80 \%$ \\
\hline North Sumatera & 214,800 & 301,369 & $3.40 \%$ & 25 & $4.60 \%$ & $5.00 \%$ \\
\hline West Sumatera & 225,440 & 365,050 & $4.80 \%$ & 17 & $1.80 \%$ & $1.90 \%$ \\
\hline Riau & 301,760 & 535,274 & $5.70 \%$ & 10 & $2.30 \%$ & $3.10 \%$ \\
\hline Jambi & 207,052 & 310,820 & $4.10 \%$ & 19 & $1.10 \%$ & $1.30 \%$ \\
\hline South Sumatera & 184,783 & 313,857 & $5.30 \%$ & 15 & $2.80 \%$ & $3.00 \%$ \\
\hline Bengkulu & 191,671 & 257,334 & $2.90 \%$ & 28 & $0.60 \%$ & $0.70 \%$ \\
\hline Lampung & 166,901 & 247,997 & $4.00 \%$ & 22 & $2.80 \%$ & $3.20 \%$ \\
\hline Bangka Belitung & 257,159 & 365,442 & $3.50 \%$ & 24 & $0.40 \%$ & $0.50 \%$ \\
\hline Jakarta & 519,288 & 762,730 & $3.80 \%$ & 23 & $3.60 \%$ & $4.00 \%$ \\
\hline West Java & 220,854 & 424,052 & $6.50 \%$ & 6 & $18.90 \%$ & $19.00 \%$ \\
\hline Central Java & 180,000 & 310,983 & $5.50 \%$ & 12 & $15.70 \%$ & $13.90 \%$ \\
\hline Yogyakarta & 270,803 & 381,836 & $3.40 \%$ & 26 & $1.80 \%$ & $1.70 \%$ \\
\hline East Java & 182,337 & 322,176 & $5.70 \%$ & 11 & $19.00 \%$ & $16.30 \%$ \\
\hline Banten & 256,943 & 465,126 & $5.90 \%$ & 8 & $3.70 \%$ & $4.30 \%$ \\
\hline Bali & 303,913 & 608,274 & $6.90 \%$ & 4 & $1.80 \%$ & $1.70 \%$ \\
\hline West Nusa Tenggara & 166,588 & 283,427 & $5.30 \%$ & 14 & $1.80 \%$ & $2.00 \%$ \\
\hline East Nusa Tenggara & 151,452 & 213,074 & $3.40 \%$ & 27 & $1.50 \%$ & $1.70 \%$ \\
\hline West Kalimantan & 205,660 & 349,965 & $5.30 \%$ & 13 & $1.50 \%$ & $1.70 \%$ \\
\hline Central Kalimantan & 221,695 & 414,965 & $6.30 \%$ & 7 & $0.80 \%$ & $1.00 \%$ \\
\hline South Kalimantan & 227,564 & 380,050 & $5.10 \%$ & 16 & $1.50 \%$ & $1.70 \%$ \\
\hline East Kalimantan & 350,281 & 465,455 & $2.80 \%$ & 29 & $1.20 \%$ & $1.50 \%$ \\
\hline North Sulawesi & 231,784 & 490,653 & $7.50 \%$ & 2 & $1.10 \%$ & $0.90 \%$ \\
\hline Central Sulawesi & 196,332 & 309,442 & $4.50 \%$ & 18 & $0.90 \%$ & $1.10 \%$ \\
\hline South Sulawesi & 189,354 & 382,604 & $7.00 \%$ & 3 & $3.20 \%$ & $3.50 \%$ \\
\hline South East Sulawesi & 184,198 & 328,198 & $5.80 \%$ & 9 & $0.70 \%$ & $0.90 \%$ \\
\hline Gorontalo & 167,087 & 328,311 & $6.80 \%$ & 5 & $0.40 \%$ & $0.40 \%$ \\
\hline Maluku & 210,947 & 316,725 & $4.10 \%$ & 21 & $0.40 \%$ & $0.50 \%$ \\
\hline North Maluku & 149,115 & 340,475 & $8.30 \%$ & 1 & $1.30 \%$ & $0.40 \%$ \\
\hline Papua & 231,109 & 352,835 & $4.20 \%$ & 20 & $1.00 \%$ & $1.50 \%$ \\
\hline Indonesia & 217,178 & 377,946 & $5.50 \%$ & & & \\
\hline Region & 2004 & 2014 & Growth rate & & & \\
\hline Sumatra & 213,334 & 333,143 & $4.50 \%$ & 5 & $18.10 \%$ & $20.40 \%$ \\
\hline Java-Bali & 222,101 & 400,204 & $5.90 \%$ & 2 & $64.60 \%$ & $60.80 \%$ \\
\hline Kalimantan & 248,397 & 399,296 & $4.70 \%$ & 4 & $5.00 \%$ & $5.90 \%$ \\
\hline Sulawesi & 195,543 & 375,740 & $6.50 \%$ & 1 & $6.30 \%$ & $6.80 \%$ \\
\hline East Indonesia & 173,312 & 287,968 & $5.10 \%$ & 3 & $6.10 \%$ & $6.10 \%$ \\
\hline Indonesia & 217,178 & 377,946 & $5.50 \%$ & & & \\
\hline
\end{tabular}

Calculated from Susenas 2004 and 2014. 


\section{Redistribution}

If there is no change in expenditure inequality, economic growth should reduce the incidence of poverty (i.e., headcount ratio). However, economic growth is usually associated with the change in expenditure inequality. When economic growth is accompanied by rising inequality, then the reduction of poverty will be lessened. To analyze the extent to which economic growth was conducive to the reduction of poverty, it is necessary to know whether economic growth was accompanied by rising expenditure inequality or not. Tables 3 and 4 present expenditure inequality, respectively, in 2004 and 2014, where inequality is measured by the Theil $\mathrm{L}$ and $\mathrm{T}$ indices and the Gini coefficient. ${ }^{3}$ These tables present also the result of a decomposition analysis by provinces using the Theil $\mathrm{L}$ and $\mathrm{T}$ indices. $^{4}$ As measured by the Gini coefficient, expenditure inequality was 0.34

${ }^{3}$ The Gini coefficient is obtained by the following formula.

$G=\frac{2}{n \mu} \operatorname{cov}\left(i, y_{i}\right)$ where $\mathrm{n}$ is total number of households, $\mu$ is the mean per capita expenditure, $y_{i}$ is per capita expenditure of household $i$, and $\operatorname{cov}(a, b)$ is the covariance between variables $a$ and $b$. The Gini coefficient ranges between 0 (perfect equality) and 1 (perfect inequality). in 2004 in Indonesia, but has increased notably to 0.43 in 2014 .

\section{Economic Growth, Redistribution and}

\section{Poverty Reduction: Pro-poorness of}

\section{EconomicGrowth}

As discussed in the previous section, economic growth should reduce the incidence of poverty if there is no change in expenditure inequality. However, economic growth is usually associated with the change in expenditure inequality. When economic growth is accompanied by declining inequality, then it is said to be pro-poor. On the other hand, when economic growth is accompanied by rising inequality, it is not pro-poor. If rising inequality does not wholly offset the poverty-reducing effect of economic growth, however, the incidence of poverty will decrease even though the growth is not strictly pro-poor.It will analyze the propoorness of economic growth for each province. Since most Indonesian provinces experienced a rise in expenditure

${ }^{4}$ Theil indices can be decomposed additively into the within-province and between-province inequality components $\left(L_{W}\right.$ and $L_{B}$ or $T_{W}$ and $\left.T_{B}\right)$ as follows:

$L=\frac{1}{n} \sum_{i=1}^{30} \sum_{j=1}^{n_{i}} \ln \left(\frac{\mu}{y_{i j}}\right)=L_{W}+L_{B}$ and $\quad T=$ $\frac{1}{n} \sum_{l=1}^{30} \sum_{j=1}^{n_{i}} \frac{y_{i j}}{\mu} \ln \left(\frac{y_{i j}}{\mu}\right)=T_{W}+T_{B}$

where $n$ is total number of households, $\mu$ is the mean per capita expenditure and $y_{i j}$ is per capita expenditure of household $j$ in province $i$. 
Spatial Dimensions of Economic Growth, Redistribution, and Poverty Reduction During the Yudhoyono Period in Indonesia

Email:witri.maji@gmail.com

inequality, their economic growth was not strictly pro-poor. Nonetheless, they achieved the reduction of the incidence of poverty, since rising inequality did not wholly offset the poverty-reducing effect of economic growth. Therefore, this section investigates relative pro-poorness of economic growth.

Table 3. Inequality in Per Capita Expenditure in 2004

\begin{tabular}{|c|c|c|c|c|c|}
\hline Province & Theil L & Contribution & Theil T & Contribution & Gini \\
\hline Aceh & 0.141 & $1.3 \%$ & 0.155 & $1.0 \%$ & 0.294 \\
\hline North Sumatera & 0.132 & $3.3 \%$ & 0.152 & $3.0 \%$ & 0.283 \\
\hline West Sumatera & 0.150 & $1.5 \%$ & 0.169 & $1.4 \%$ & 0.304 \\
\hline Riau & 0.175 & $2.2 \%$ & 0.202 & $2.8 \%$ & 0.328 \\
\hline Jambi & 0.121 & $0.7 \%$ & 0.136 & $0.6 \%$ & 0.271 \\
\hline South Sumatera & 0.123 & $1.8 \%$ & 0.134 & $1.4 \%$ & 0.275 \\
\hline Bengkulu & 0.143 & $0.5 \%$ & 0.164 & $0.4 \%$ & 0.298 \\
\hline Lampung & 0.142 & $2.2 \%$ & 0.159 & $1.5 \%$ & 0.295 \\
\hline Bangka Belitung & 0.119 & $0.3 \%$ & 0.127 & $0.3 \%$ & 0.270 \\
\hline Jakarta & 0.251 & $4.9 \%$ & 0.354 & $13.3 \%$ & 0.385 \\
\hline West Java & 0.144 & $14.7 \%$ & 0.167 & $13.9 \%$ & 0.295 \\
\hline Central Java & 0.133 & $11.3 \%$ & 0.155 & $8.8 \%$ & 0.287 \\
\hline Yogyakarta & 0.282 & $2.8 \%$ & 0.319 & $3.1 \%$ & 0.420 \\
\hline East Java & 0.156 & $16.0 \%$ & 0.197 & $13.5 \%$ & 0.309 \\
\hline Banten & 0.163 & $3.2 \%$ & 0.191 & $3.6 \%$ & 0.314 \\
\hline Bali & 0.146 & $1.4 \%$ & 0.145 & $1.6 \%$ & 0.294 \\
\hline West Nusa Tenggara & 0.138 & $1.3 \%$ & 0.157 & $0.9 \%$ & 0.292 \\
\hline East Nusa Tenggara & 0.156 & $1.3 \%$ & 0.173 & $0.8 \%$ & 0.311 \\
\hline West Kalimantan & 0.156 & $1.3 \%$ & 0.181 & $1.1 \%$ & 0.308 \\
\hline Central Kalimantan & 0.129 & $0.6 \%$ & 0.140 & $0.5 \%$ & 0.282 \\
\hline South Kalimantan & 0.148 & $1.2 \%$ & 0.164 & $1.1 \%$ & 0.302 \\
\hline East Kalimantan & 0.237 & $1.5 \%$ & 0.317 & $2.6 \%$ & 0.377 \\
\hline North Sulawesi & 0.121 & $0.7 \%$ & 0.130 & $0.6 \%$ & 0.274 \\
\hline Central Sulawesi & 0.166 & $0.8 \%$ & 0.210 & $0.7 \%$ & 0.318 \\
\hline South Sulawesi & 0.161 & $2.8 \%$ & 0.188 & $2.3 \%$ & 0.314 \\
\hline South East Sulawesi & 0.134 & $0.5 \%$ & 0.142 & $0.4 \%$ & 0.286 \\
\hline Gorontalo & 0.143 & $0.3 \%$ & 0.151 & $0.2 \%$ & 0.296 \\
\hline Maluku & 0.145 & $0.3 \%$ & 0.166 & $0.3 \%$ & 0.296 \\
\hline North Maluku & 0.034 & $0.2 \%$ & 0.044 & $0.2 \%$ & 0.099 \\
\hline Papua & 0.200 & $1.1 \%$ & 0.213 & $1.0 \%$ & 0.348 \\
\hline Within-province & 0.152 & $81.8 \%$ & 0.191 & $82.7 \%$ & \\
\hline Between-province & 0.034 & $18.2 \%$ & 0.040 & $17.3 \%$ & \\
\hline Indonesia & 0.185 & $100.0 \%$ & 0.231 & $100.0 \%$ & 0.337 \\
\hline Region & Theil L & Contribution & Theil T & Contribution & Gini \\
\hline Sumatra & 0.155 & $15.2 \%$ & 0.176 & $13.6 \%$ & 0.308 \\
\hline Java-Bali & 0.196 & $68.4 \%$ & 0.251 & $71.7 \%$ & 0.347 \\
\hline Kalimantan & 0.191 & $5.1 \%$ & 0.239 & $5.9 \%$ & 0.340 \\
\hline Sulawesi & 0.154 & $5.2 \%$ & 0.176 & $4.3 \%$ & 0.308 \\
\hline East Indonesia & 0.145 & $4.8 \%$ & 0.167 & $3.5 \%$ & 0.295 \\
\hline Within-region & 0.183 & $98.7 \%$ & 0.229 & $99.0 \%$ & \\
\hline Between-region & 0.002 & $1.3 \%$ & 0.002 & $1.0 \%$ & \\
\hline Indonesia & 0.185 & $100.0 \%$ & 0.231 & $100.0 \%$ & 0.337 \\
\hline
\end{tabular}

(Source) Calculated from Susenas 2004 and 2014. 
Table 4. Inequality in Per Capita Expenditure in 2014 (at 2004 Constant Prices)

\begin{tabular}{|c|c|c|c|c|c|}
\hline Province & Theil L & Contribution & Theil T & Contribution & Gini \\
\hline Aceh & 0.189 & $1.2 \%$ & 0.217 & $0.8 \%$ & 0.343 \\
\hline North Sumatera & 0.175 & $2.9 \%$ & 0.202 & $2.3 \%$ & 0.329 \\
\hline West Sumatera & 0.208 & $1.3 \%$ & 0.241 & $1.2 \%$ & 0.359 \\
\hline Riau & 0.255 & $2.7 \%$ & 0.289 & $3.6 \%$ & 0.398 \\
\hline Jambi & 0.191 & $0.8 \%$ & 0.232 & $0.7 \%$ & 0.343 \\
\hline South Sumatera & 0.267 & $2.7 \%$ & 0.316 & $2.2 \%$ & 0.406 \\
\hline Bengkulu & 0.237 & $0.6 \%$ & 0.281 & $0.4 \%$ & 0.383 \\
\hline Lampung & 0.212 & $2.3 \%$ & 0.263 & $1.6 \%$ & 0.361 \\
\hline Bangka Belitung & 0.174 & $0.3 \%$ & 0.215 & $0.3 \%$ & 0.326 \\
\hline Jakarta & 0.282 & $3.8 \%$ & 0.327 & $7.5 \%$ & 0.415 \\
\hline West Java & 0.296 & $19.0 \%$ & 0.354 & $21.4 \%$ & 0.426 \\
\hline Central Java & 0.253 & $11.9 \%$ & 0.311 & $10.1 \%$ & 0.395 \\
\hline Yogyakarta & 0.291 & $1.7 \%$ & 0.334 & $1.6 \%$ & 0.423 \\
\hline East Java & 0.251 & $13.9 \%$ & 0.313 & $12.4 \%$ & 0.393 \\
\hline Banten & 0.284 & $4.1 \%$ & 0.324 & $4.8 \%$ & 0.417 \\
\hline Bali & 0.298 & $1.7 \%$ & 0.329 & $2.6 \%$ & 0.422 \\
\hline West Nusa Tenggara & 0.284 & $2.0 \%$ & 0.340 & $1.5 \%$ & 0.419 \\
\hline East Nusa Tenggara & 0.240 & $1.3 \%$ & 0.305 & $0.8 \%$ & 0.385 \\
\hline West Kalimantan & 0.300 & $1.7 \%$ & 0.346 & $1.6 \%$ & 0.429 \\
\hline Central Kalimantan & 0.235 & $0.8 \%$ & 0.263 & $0.8 \%$ & 0.381 \\
\hline South Kalimantan & 0.218 & $1.2 \%$ & 0.255 & $1.2 \%$ & 0.365 \\
\hline East Kalimantan & 0.199 & $1.0 \%$ & 0.234 & $1.3 \%$ & 0.350 \\
\hline North Sulawesi & 0.312 & $1.0 \%$ & 0.341 & $1.2 \%$ & 0.435 \\
\hline Central Sulawesi & 0.264 & $0.9 \%$ & 0.342 & $0.8 \%$ & 0.402 \\
\hline South Sulawesi & 0.328 & $3.9 \%$ & 0.383 & $3.8 \%$ & 0.445 \\
\hline South East Sulawesi & 0.283 & $0.8 \%$ & 0.325 & $0.7 \%$ & 0.416 \\
\hline Gorontalo & 0.371 & $0.5 \%$ & 0.418 & $0.4 \%$ & 0.470 \\
\hline Maluku & 0.210 & $0.4 \%$ & 0.227 & $0.3 \%$ & 0.360 \\
\hline North Maluku & 0.274 & $0.3 \%$ & 0.316 & $0.3 \%$ & 0.412 \\
\hline Papua & 0.318 & $1.6 \%$ & 0.343 & $1.4 \%$ & 0.439 \\
\hline Within-province & 0.261 & $88.5 \%$ & 0.315 & $89.5 \%$ & \\
\hline Between-province & 0.034 & $11.5 \%$ & 0.037 & $10.5 \%$ & \\
\hline Total & 0.295 & $100.0 \%$ & 0.352 & $100.0 \%$ & 0.425 \\
\hline Region & & Contribution & & Contribution & \\
\hline Sumatra & 0.246 & $17.0 \%$ & 0.290 & $14.8 \%$ & 0.389 \\
\hline Java-Bali & 0.306 & $63.1 \%$ & 0.368 & $67.3 \%$ & 0.433 \\
\hline Kalimantan & 0.246 & $4.9 \%$ & 0.280 & $4.9 \%$ & 0.387 \\
\hline Sulawesi & 0.322 & $7.4 \%$ & 0.375 & $7.2 \%$ & 0.442 \\
\hline East Indonesia & 0.292 & $6.0 \%$ & 0.339 & $4.5 \%$ & 0.425 \\
\hline Within-region & 0.291 & $98.4 \%$ & 0.347 & $98.7 \%$ & \\
\hline Between-region & 0.005 & $1.6 \%$ & 0.005 & $1.3 \%$ & \\
\hline Total & 0.295 & $100.0 \%$ & 0.352 & $100.0 \%$ & 0.425 \\
\hline
\end{tabular}

(Source) Calculated from Susenas 2004 and 2014.

In the period from 2004 to 2014 , Indonesia grew at $5.5 \%$ in terms of mean per capita expenditure (see Table 2) and achieved a substantial reduction in the incidence of poverty (see Table 1). However, it saw a rise in expenditure inequality; thus, the 
growth was not pro-poor in Indonesia.

Figure 1 exhibits the growth incidence curve for Indonesia. An upward sloping curve indicates Indonesian economic growth was not pro-poor, since poorer households grew at much smaller rate than the national average.

Figure 1. Growth Incidence Curve for Indonesia

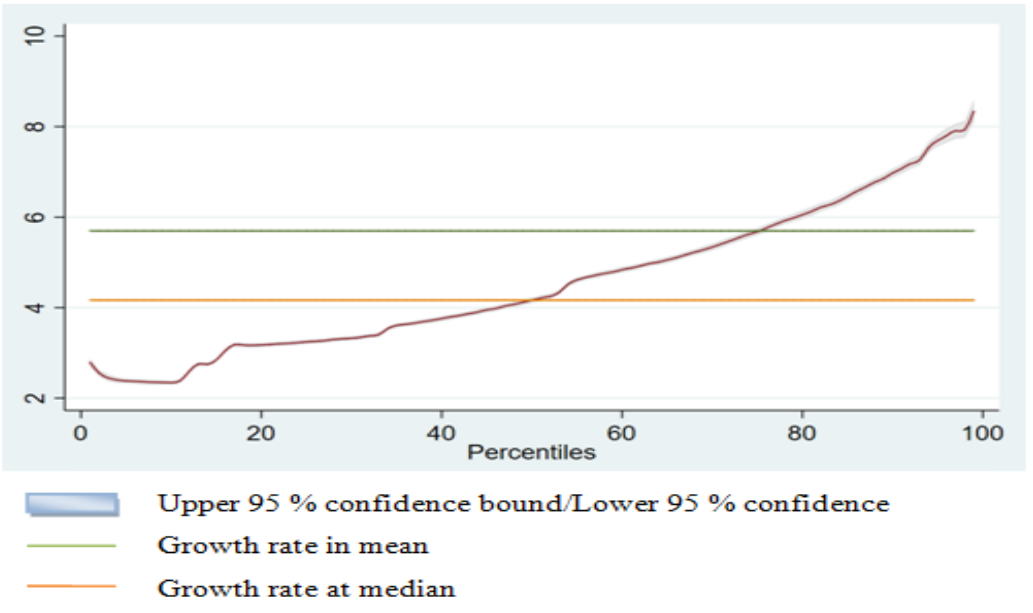

Source: Estimated from SUSENAS 2004 and 2014

As shown in Table 5, West

Sumatera and Bangka Belitung recorded a very large decrease in the incidence of poverty, though their growth rates were not large. This is due to relatively smallincrease in expenditure inequality. Jakarta also grew less rapidly, but its inequality rose only slightly and thus the incidence of poverty has declined by 6.2 percentage points.

There is a large variation among provinces in terms of pro-poorness of economic growth. Employing the classification described above, provinces are classified into the following five groups. The five groups are classified in:
- $\quad$ PPGI $<0$, growth is anti poor North Maluku

- $\quad 0<$ PPGI $\leq 0.33$, growth is weakly pro-poor Gorontalo, \& Bengkulu

- $0.33<$ PPGI $\leq 0.66$, growth is moderately pro-poor Aceh, North Sumatera, Jambi, South Sumatera, West Java, Banten, Central Java, East Java, Bali, West Kalimantan, Central Kalimantan, South East Sulawesi, North Sulawesi,South Sulawesi, Central Sulawesi, East Nusa Tenggara, West Nusa Tenggara, Maluku

- $0.66<$ PPGI $<1.0$, growth is propoor Riau, West Sumatera, Bangka 
Spatial Dimensions of Economic Growth, Redistribution, and Poverty Reduction During the Yudhoyono Period in Indonesia

Email:witri.maji@gmail.com

Belitung, Jakarta, South

Kalimantan

- $\quad$ PPGI $\geq 1.0$, growth is highly pro-

poor Yogyakarta, East Kalimantan,

Papua.

Table 5. Decomposition of Change in Poverty into Growth and Redistribution

\begin{tabular}{|c|c|c|c|c|c|c|}
\hline Province & $\begin{array}{r}\text { Poverty } \\
\text { in } \\
2004 \\
(1)\end{array}$ & $\begin{array}{r}\text { Poverty } \\
\text { in } \\
2014 \\
(2)\end{array}$ & $\begin{array}{r}\text { Change in } \\
\text { poverty } \\
(3)=(2)-(1) \\
=(\mathrm{GE})+(\mathrm{IE})\end{array}$ & $\begin{array}{r}\text { Change in } \\
\text { poverty due } \\
\text { to growth } \\
(\mathrm{GE})\end{array}$ & $\begin{array}{r}\text { Change in } \\
\text { poverty due } \\
\text { to } \\
\text { redistribution } \\
\text { (IE) }\end{array}$ & $\begin{array}{r}\text { Annual } \\
\text { average rate } \\
\text { of change in } \\
\text { poverty }\end{array}$ \\
\hline Aceh & 26.0 & 16.3 & -9.6 & -16.9 & 7.3 & -4.6 \\
\hline North Sumatera & 18.0 & 7.7 & -10.3 & -16.2 & 5.9 & -8.5 \\
\hline West Sumatera & 26.5 & 6.6 & -19.9 & -27.3 & 7.3 & -13.9 \\
\hline Riau & 26.0 & 6.6 & -19.3 & -28.1 & 8.8 & -13.6 \\
\hline Jambi & 19.9 & 8.0 & -11.9 & -21.2 & 9.3 & -9.1 \\
\hline South Sumatera & 24.4 & 12.7 & -11.7 & -27.1 & 15.4 & -6.5 \\
\hline Bengkulu & 20.6 & 16.5 & -4.1 & -17.5 & 13.4 & -2.2 \\
\hline Lampung & 32.2 & 13.9 & -18.4 & -27.8 & 9.4 & -8.4 \\
\hline Bangka Belitung & 18.5 & 5.7 & -12.8 & -18.0 & 5.2 & -11.7 \\
\hline Jakarta & 9.1 & 3.0 & -6.2 & -10.7 & 4.5 & -11.2 \\
\hline West Java & 22.1 & 8.4 & -13.6 & -29.2 & 15.6 & -9.6 \\
\hline Central Java & 35.4 & 13.4 & -22.0 & -34.7 & 12.7 & -9.7 \\
\hline Yogyakarta & 29.4 & 11.9 & -17.5 & -17.3 & -0.2 & -9.1 \\
\hline East Java & 39.1 & 11.5 & -27.6 & -36.8 & 9.3 & -12.2 \\
\hline Banten & 14.7 & 4.8 & -9.9 & -21.8 & 11.9 & -11.2 \\
\hline Bali & 14.4 & 3.7 & -10.6 & -21.7 & 11.1 & -13.4 \\
\hline West Nusa Tenggara & 32.0 & 15.8 & -16.2 & -31.7 & 15.6 & -7.0 \\
\hline East Nusa Tenggara & 31.6 & 16.3 & -15.3 & -25.0 & 9.7 & -6.6 \\
\hline West Kalimantan & 17.5 & 7.9 & -9.6 & -23.2 & 13.6 & -8.0 \\
\hline Central Kalimantan & 20.6 & 5.4 & -15.2 & -25.7 & 10.6 & -13.3 \\
\hline South Kalimantan & 16.0 & 3.8 & -12.2 & -19.2 & 7.0 & -14.4 \\
\hline East Kalimantan & 20.1 & 5.7 & -14.4 & -11.9 & -2.6 & -12.7 \\
\hline North Sulawesi & 17.9 & 7.3 & -10.6 & -26.9 & 16.4 & -8.9 \\
\hline Central Sulawesi & 28.2 & 12.0 & -16.1 & -27.1 & 10.9 & -8.5 \\
\hline South Sulawesi & 25.2 & 9.0 & -16.2 & -30.6 & 14.4 & -10.3 \\
\hline South East Sulawesi & 24.0 & 11.0 & -13.0 & -27.9 & 14.9 & -7.8 \\
\hline Gorontalo & 23.4 & 16.0 & -7.3 & -27.9 & 20.6 & -3.8 \\
\hline Maluku & 23.7 & 13.2 & -10.5 & -21.2 & 10.7 & -5.9 \\
\hline North Maluku & 4.2 & 7.2 & 3.0 & -28.8 & 31.8 & 5.4 \\
\hline Papua & 49.4 & 22.9 & -26.5 & -21.4 & -5.1 & -7.7 \\
\hline Indonesia & 27.3 & 10.0 & -17.3 & -29.2 & 11.9 & -10.0 \\
\hline \multicolumn{7}{|l|}{ Region } \\
\hline Sumatra & 24.0 & 10.2 & -13.8 & -24.4 & 10.6 & -8.6 \\
\hline Java-Bali & 29.2 & 9.7 & -19.4 & -31.2 & 11.7 & -11.0 \\
\hline Kalimantan & 18.2 & 5.7 & -12.4 & -19.7 & 7.3 & -11.5 \\
\hline Sulawesi & 24.1 & 9.9 & -14.2 & -29.4 & 15.2 & -8.9 \\
\hline East Indonesia & 28.2 & 16.9 & -11.3 & -27.3 & 16.0 & -5.1 \\
\hline Indonesia & 27.3 & 10.0 & -17.3 & -29.2 & 11.9 & -10.0 \\
\hline
\end{tabular}

(Source) Calculated from Susenas 2004 and 2014. 
Table 6. Pro-poor Growth Indices

\begin{tabular}{|c|c|c|c|c|c|}
\hline Province & $\begin{array}{r}\text { Annual } \\
\text { average } \\
\text { growth rate } \\
\text { of mean } \\
\text { per capita } \\
\text { expenditure } \\
(1) \\
\end{array}$ & $\begin{array}{r}\text { Growth } \\
\text { elasticity } \\
\text { of poverty } \\
(2)\end{array}$ & $\begin{array}{r}\text { Growth } \\
\text { elasticity of } \\
\text { poverty without } \\
\text { redistribution } \\
\text { (3) }\end{array}$ & $\begin{array}{r}\text { PPGI } \\
(\text { Kakwani\&Pernia) } \\
(4)=(2) /(3)\end{array}$ & $\begin{array}{r}\text { PEGR } \\
(\text { Kakwani\& } \\
\text { Son) } \\
(5)=(4) \times(1)\end{array}$ \\
\hline Aceh & $2.5 \%$ & -1.86 & -3.20 & 0.58 & $1.4 \%$ \\
\hline North Sumatera & $3.4 \%$ & -2.50 & -3.97 & 0.63 & $2.1 \%$ \\
\hline West Sumatera & $4.8 \%$ & -2.89 & -3.88 & 0.75 & $3.6 \%$ \\
\hline Riau & $5.7 \%$ & -2.38 & -3.51 & 0.68 & $3.9 \%$ \\
\hline Jambi & $4.1 \%$ & -2.24 & -3.81 & 0.59 & $2.4 \%$ \\
\hline South Sumatera & $5.3 \%$ & -1.23 & -3.25 & 0.38 & $2.0 \%$ \\
\hline Bengkulu & $2.9 \%$ & -0.75 & -3.63 & 0.21 & $0.6 \%$ \\
\hline Lampung & $4.0 \%$ & -2.13 & -3.27 & 0.65 & $2.6 \%$ \\
\hline Bangka Belitung & $3.5 \%$ & -3.34 & -4.42 & 0.76 & $2.7 \%$ \\
\hline Jakarta & $3.8 \%$ & -2.92 & -4.22 & 0.69 & $2.7 \%$ \\
\hline West Java & $6.5 \%$ & -1.47 & -3.52 & 0.42 & $2.7 \%$ \\
\hline Central Java & $5.5 \%$ & -1.77 & -3.88 & 0.46 & $2.5 \%$ \\
\hline Yogyakarta & $3.4 \%$ & -2.63 & -2.37 & 1.11 & $3.8 \%$ \\
\hline East Java & $5.7 \%$ & -2.15 & -3.69 & 0.58 & $3.3 \%$ \\
\hline Banten & $5.9 \%$ & -1.89 & -3.84 & 0.49 & $2.9 \%$ \\
\hline Bali & $6.9 \%$ & -1.94 & -3.99 & 0.49 & $3.4 \%$ \\
\hline West Nusa Tenggara & $5.3 \%$ & -1.32 & -3.09 & 0.43 & $2.3 \%$ \\
\hline East Nusa Tenggara & $3.4 \%$ & -1.95 & -3.24 & 0.60 & $2.1 \%$ \\
\hline West Kalimantan & $5.3 \%$ & -1.50 & -3.75 & 0.40 & $2.1 \%$ \\
\hline Central Kalimantan & $6.3 \%$ & -2.12 & -3.87 & 0.55 & $3.4 \%$ \\
\hline South Kalimantan & $5.1 \%$ & -2.81 & -4.09 & 0.69 & $3.5 \%$ \\
\hline East Kalimantan & $2.8 \%$ & -4.46 & -3.63 & 1.23 & $3.5 \%$ \\
\hline North Sulawesi & $7.5 \%$ & -1.19 & -3.45 & 0.35 & $2.6 \%$ \\
\hline Central Sulawesi & $4.5 \%$ & -1.87 & -3.55 & 0.53 & $2.4 \%$ \\
\hline South Sulawesi & $7.0 \%$ & -1.47 & -3.39 & 0.43 & $3.0 \%$ \\
\hline South East Sulawesi & $5.8 \%$ & -1.35 & -3.29 & 0.41 & $2.4 \%$ \\
\hline Gorontalo & $6.8 \%$ & -0.56 & -2.79 & 0.20 & $1.3 \%$ \\
\hline Maluku & $4.1 \%$ & -1.45 & -3.29 & 0.44 & $1.8 \%$ \\
\hline North Maluku & $8.3 \%$ & 0.65 & -4.28 & -0.15 & $-1.3 \%$ \\
\hline Papua & $4.2 \%$ & -1.82 & -1.38 & 1.32 & $5.6 \%$ \\
\hline Indonesia & $5.5 \%$ & -1.81 & -3.52 & 0.51 & $2.7 \%$ \\
\hline \multicolumn{6}{|l|}{ Region } \\
\hline Sumatra & $4.5 \%$ & -1.93 & -3.49 & 0.55 & $2.5 \%$ \\
\hline Java-Bali & $5.9 \%$ & -1.86 & -3.70 & 0.50 & $3.0 \%$ \\
\hline Kalimantan & $4.7 \%$ & -2.43 & -3.77 & 0.64 & $3.1 \%$ \\
\hline Sulawesi & $6.5 \%$ & -1.36 & -3.41 & 0.40 & $2.6 \%$ \\
\hline East Indonesia & $5.1 \%$ & -1.00 & -2.39 & 0.42 & $2.1 \%$ \\
\hline Indonesia & $5.5 \%$ & -1.81 & -3.52 & 0.51 & $2.7 \%$ \\
\hline
\end{tabular}

(Source) Calculated from Susenas 2004 and 2014. 


\section{CONCLUSION}

Based on the 2004 and 2014 National Socio-Economic Surveys (Susenas), this study attempted to analyze the relationship between economic growth, redistribution and poverty reduction from a spatial perspective in Indonesia during the Yudhoyono period from 2004 to 2014 using the poverty decomposition method, the growth incidence curve and pro-poor growth indices. The following provides a summary of findings. First, in the period from 2004 to 2014 , Indonesia grew at $5.5 \%$ and achieved a substantialreduction in the incidence of poverty from $27 \%$ to $10 \%$. However, it experienced a rise in expenditure inequality. Though economic growth reduced the incidence of poverty, the growth was not pro-poor since the rise in inequality lessened the poverty reducing effect of economic growth as indicated by the upward sloping growth incidence curve. Second, all regions, i.e., Sumatra, JavaBali, Kalimantan, Sulawesi and East Indonesia, had an upward sloping growth incidence curve and their pro-poor growth index (PPGI) ranged between 0.40 and 0.64. In other words, their economic growth was not strictly pro-poor. However, the shape of the growth incidence curve differs between regions, reflecting the difference in economic growth and redistribution.

Third, there is a large variation among provinces in the pro-poorness of economic growth. Using the classification proposed by Kakwani and Pernia(2000), provinces can be classified into the following five groups with respect to the pro-poor growth index (PPGI): (1) PPGI < 0 , growth is antipoor;(2) $0<\mathrm{PPGI} \leq 0.33$, growth is weakly pro-poor;(3) $0.33<$ PPGI $\leq 0.66$, growth is moderately pro-poor;(4) $0.66<$ PPGI < 1.0, growth is pro-poor; and(5) PPGI $\geq 1.0$, growth is highly propoor.Out of 30 provinces, 19 provinces are placed in group (3). Among the other 11 provinces, only North Maluku is in group (3), as its PPGI is negative. North Maluku registered an increase in the incidence of poverty, due to a large increase in expenditure inequality. Fourth, Gorontalo and Bengkulu are placed in group (2). Like North Maluku, Gorontalo achieved a very high growth, but the reduction of poverty is very small due to a large increase in inequality. While Bengkulu had a relatively small increase in inequality, its growth was also small and thus, the incidence of poverty has declined only slightly.

Fifth, Riau, West Sumatra, Bangka Belitung, Jakarta and South Kalimantan are 
in group (4) and their economic growth is pro-poor. Among provinces in group (4), Riau, West Sumatera and Bangka Belitung are Sumatra provinces. West Sumatera and Bangka Belitung recorded a very large decrease in the incidence of poverty, though their growth rates were not large. This is due to relatively small increase in expenditure inequality. Jakarta also grew less rapidly, but its inequality rose only slightly and thus the incidence of poverty has declined. Sixth, Yogyakarta, East Kalimantan and Papua are in group (5) and achieved highly pro-poor growth, as their PPGI exceeds one. East Kalimantan is the only province that experienced a decrease in expenditure inequality. Though its growth rate was much smaller than the national average, it reduced its incidence of poverty. The growth incidence curve is slightly downward sloping. Papua realized a large reduction in the incidence of poverty from $49 \%$ to $23 \%$. The change in poverty due to redistribution was negative in Papua, meaning that the change in expenditure inequality was conducive to the reduction of poverty. Though Yogyakarta grew at a much smaller rate than the national average, its expenditure inequality remained almost constant and thus, its PPGI exceeds one.

\section{Policy Implications}

From these findings, some policy implications can be obtained. First, economic growth is moderately pro-poor in all regions according to the criteria proposed by Kakwani and Pernia (2000). But there is a large variation in the propoorness of economic growth among provinces. To achieve a balanced pro-poor growth across provinces, the government needs to formulate policies whichtake into account differences in natural and human resources, economic activities and infrastructure.Community empowerment program such as village fund (dana desa) is one possible solution as it can promotes the development, especially in a remote area with exploring the local resources.

Second, though the provinces of Papua and West and East Nusa Tenggara realized a moderately or highly pro-poor growth, their incidence of poverty was still high in 2014. To further reduce their incidence of poverty, the government needs to introduce policies to accelerate economic growth as their growth rates were below the national average. Additionally, it should implement policies to reduce inequality. In Papua and West Papua are still doing development of toll road called trans Papua. While in East Nusa Tenggara developing 
water resource with constructing Mbay Dam started in 2018 and Kolhua Dam, in West Nusa Tenggara Meninting Dam also under construction. (Ministry of Public Works,2018).

Third, Bengkulu and Gorontalo still had a very high incidence of poverty in 2014 and its economic growth was weakly pro-poor. Though Bengkulu registered a relatively low inequality, its growth performance was very weak. The governmentshould thus introduce policies to promote economic growth for this province. On the other hand, Gorontalo achieved a very high economic growth, but its inequality has risen substantially and the province had the highest inequality in 2014.

Therefore, the government needs to strengthen redistributive policies to alleviate inequality. To enhance the economic growth of Bengkulu Province, Ministry of Public Work will construct five bridges in North Bengkulu to support neighbourhood economically (Ministry of Public Works, 2018). On the other hand, the process of developing the Gorontalo or Gorontalo Outer Ring Road (GORR) ring road entered the third segment.(Economic Bussines, 2019).

\section{ACKNOWLEDGEMENT}

Thank you to the Professor in Public Management and Administration of Rikkyo University, Tokyo Japan for the support given in carrying out this research.

\section{REFERENCES}

Akita, T. \& Lukman, R.A. 1999. Spatial Patterns of Expenditure Inequalities in Indonesia: 1987, 1990 and 1993, Bulletin of Indonesian Economic Studies, 35:2, 67-90.

Bhattacharyya, S., \& Resosudarmo, B.P. 2015. Growth, Growth Accelerations, and the Poor: Lessons from Indonesia. World Development Vol. 66, pp. 154165.

Central Bureau of Statistics. Data \& Information Poverty in 2004, Book 1 : Province.

Central Bureau of Statistics. 2018. Poverty Profile in Indonesia September 2018. Official News Statistics, No. 07/01/Th. XXII, January, 15th 2019.

Central Bureau of Statistics. 2018. Level of Inequality in Indonesian Population Expenditure September 2018. Official News Statistics, No. 08/01/Th. XXII, January, 15th 2019.

Dabla-Norris, E., K. Kochhar, N. Suphaphiphat, F. Ricka and E. Tsounta. 2015. Causes and Consequences of Income Inequality: a global perspective. 
IMF Staff Discussion Note SDN/15/13. Washington, DC: International Monetary Fund.

Datt, G., \& Ravallion, M. 1992. Growth and redistribution components of changes in poverty measures : A decomposition with applications to Brazil and India in the 1980s. Journal of development economics, 38(2), 275-295.

De Silva, I., \& Sumarto, S. 2014. Does Economic Growth Really Benefit the Poor? Income Distribution Dynamics . Bulletin of Indonesian Economic Studies.

Economic Bussines. 2019. Construction of the Gorontalo Ring Road Enter the Third Segment. Retrieved in July 27,2019. Accessed through: https://ekonomi.bisnis.com/read/ 20190514/45/922224/pembangu nan-jalan-lingkar-gorontalomasuki-segmen-ketiga

International Monetary Fund (IMF). 2014. Redistribution, Inequality, and Growth. Research Department.

Kakwani, N. 1997. On measuring growth and inequality components of poverty with application to Thailand. The University of New South Wales, Australia.

Kakwani, N., \& Pernia, E. M. 2000. What is pro-poor growth?. Asian development review, 18(1), 1-16.

Kakwani, N., \& Son, H. H. 2008. Poverty equivalent growth rate.
Review of Income and Wealth, 54(4), 643-655.

Ministry of Public Works. 2018. Priority Infrastructure Projects in Kalimantan and Nusa Tenggara are targeted to be completed. Retrieved in July 27, 2019.

https://www.pu.go.id/berita/view/1539 5/2019-proyek-infrastrukturprioritas-di-kalimantan-dan$\underline{\text { nusa-tenggara-ditargetkan- }}$ rampung

Nasrullah, A. 2019. Ministry of Village and Public Works Developing Infrastructure in Bengkulu. Retrieved in July 27, 2019. https://finance.detik.com/infrastr uktur/d-4470356/kemendespupr-keroyok-pembangunaninfrastruktur-di-bengkulu

Ravallion, M., \& Chen, S. 2003. Measuring pro-poor growth. Economics letters, 78(1), 93-99.

Suryahadi, A., Suryadarma, D., \& Sumarto, S. 2009. The effects of location and sectoral components of economic growth on poverty: Evidence from Indonesia. Journal of Development Economics, (2009) 109-117.

Suryahadi, A., Hadiwidjaja, G., \& Sumarto, S. 2012. Economic growth and poverty reduction in Indonesia before and after the Asian financial crisis. Bulletin of Indonesian Economic Studies, Vol. 48, No. 2, 2012: 209-227.

Timmer, C. P. 2004. The road to propoor growth: the Indonesian experience in regional Perspective. Bulletin of 
Spatial Dimensions of Economic Growth, Redistribution, and Poverty Reduction During the Yudhoyono Period in Indonesia

Indonesian Economic Studies, Vol. 40, No. 2, 2004: 177-207.

Van Leeuwen, B., \& Földvári, P. 2016.

The Development of Inequality and Poverty in Indonesia, 19322008. Bulletin of Indonesian Economic Studies, Vol. 52, No. 3, 2016: 379-402.

World Bank. 2016. Indonesia's Rising Divide : Why inequality is rising, why it matters and what can be done. 